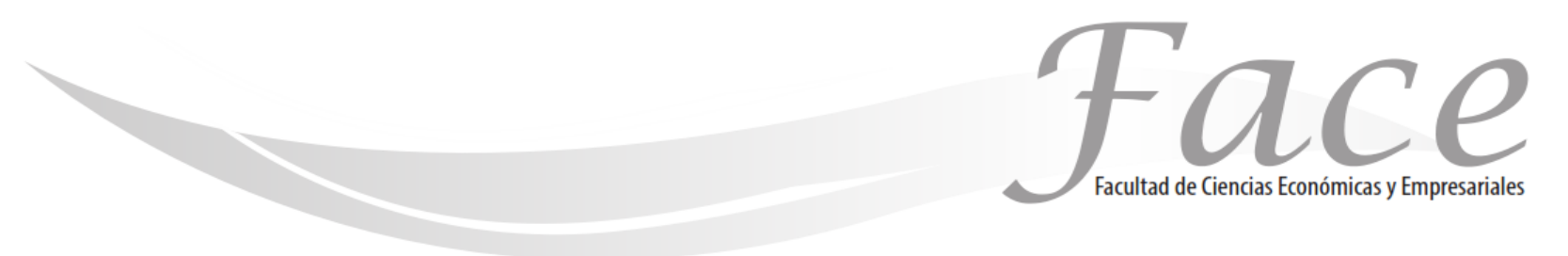

ISSN Impreso: 1794-9920

ISSN Electrónico: 2500-9338

Volumen $19-\mathrm{N}^{\circ} 2$

Año 2019

Págs. $104-114$

\title{
LA INTERNACIONALIZACIÓN PARA EL MERCADO COLOMBIANO COMO ESTRATEGIA DE INNOVACIÓN COMERCIAL DE UNA MIPE GUANAJUATENSE
}

\author{
Ana Lorena Zapata Rosas * \\ ORCID: https://orcid.org/0000-0003-1461-4962 \\ María Guadalupe Arredondo Hidalgo ** \\ ORCID: https://orcid.org/0000-0002-4971-4069
}

Fecha de Recepción: Junio 8 de 2019

Fecha de Aprobación: Septiembre10 de 2019

\section{Resumen:}

La presente investigación aborda el enfoque cualitativo desde la perspectiva de estudio de caso relacionado con una microempresa (MIPE) agroindustrial de Guanajuato. Se presume que las empresas exportadoras son más proclives a tener procesos de innovación que las llevan a ser exitosas en mercados internacionales. Se realizó el análisis de las 5 fuerzas de mercado de Michael Porter, y para la investigación del estudio de caso, se aplicó un cuestionario para medir el potencial de la empresa (Arredondo-Hidalgo, 2017). Las áreas consideradas son: a. Desarrollo del Producto, b. Eficiencia en la producción, c. Mercadotecnia Internacional y d. Gestión Gerencial. Los resultados muestran que el mercado de exportación hacia Colombia es idóneo para el tipo de producto analizado, derivado de las preferencias arancelarias del Tratado de Libre Comercio que se tiene con este país. Sin embargo, es necesario incorporar algunas adaptaciones relacionadas al gusto del producto, así como del diseño y la presentación hacia este mercado, atendiendo también a la parte de sustentabilidad y formas de expansión internacional.

Palabras clave: internacionalización, microempresas exportadoras, Colombia.

*Estudiante de la Licenciatura en Comercio Internacional por la Universidad de Guanajuato . Contacto: mg.arredondohidalgo@ugto.mx

* * Profesora Investigadora de tiempo completo de la División de Ciencias Económico Administrativas del Departamento de Gestión y Dirección de Empresas

Campus Guanajuato, de la Universidad de Guanajuato. Contacto: mg.arredondohidalgo@ugto.mx 


\title{
INTERNATIONALIZATION FOR THE COLOMBIAN MARKET AS A TRADE INNOVACIÓN STRATEGY FOR A MIPE GUANAJUATENSE
}

\begin{abstract}
:
This research addresses the qualitative approach from the perspective of a case study related to an agro-industrial micro enterprise of Guanajuato. Exporting companies are presumed to be more prone to innovation processes that lead to success in international markets. The analysis of Porter's 5 forces model was carried out, and for the investigation of the case study, a questionnaire was applied to measure the company's potential (Arredondo-Hidalgo, 2017). The areas considered are: a. Product Development, b. Production Efficiency, c. International Marketing and d. Management. The results show that the export market to Colombia is suitable for the type of product analyzed, derived from the tariff preferences of the Free Trade Agreement that is held with this country. However, it is necessary to incorporate some adaptations related to the taste of the product, as well as the design and presentation towards this market, considering the topic of sustainability and strategies of international expansion.
\end{abstract}

Keywords: internationalization, micro enterprise, Colombia.

\section{INTERNACIONALIZAÇÃO PARA O MERCADO COLOMBIANO COMO ESTRATÉGIA DE INOVAÇÃO COMERCIAL PARA UMA GUANAJUATENSE MIPE}

Resumo:

Esta pesquisa aborda a abordagem qualitativa na perspectiva de um estudo de caso relacionado a uma microempresa agroindustrial (MIPE) de Guanajuato. Presume-se que as empresas exportadoras tenham maior probabilidade de ter processos de inovação que as levem a ter sucesso nos mercados internacionais. Foi realizada a análise das cinco forças de mercado de Michael Porter e, para a investigação do estudo de caso, foi aplicado um questionário para medir o potencial da empresa (Arredondo-Hidalgo, 2017). As áreas consideradas são: a. Desenvolvimento de Produto, b. Eficiência na produção, c. Marketing internacional e d. Gerenciamento Gerenciamento Os resultados mostram que o mercado de exportação para a Colômbia é adequado ao tipo de produto analisado, derivado das preferências tarifárias do Acordo de Livre Comércio com este país. No entanto, é necessário incorporar algumas adaptações relacionadas ao sabor do produto, bem como ao design e apresentação desse mercado, considerando também a parte da sustentabilidade e as formas de expansão internacional.

Palavras-chave: internacionalização, microempresas exportadoras, Colômbia. 


\section{INTRODUCCIÓN:}

Las relaciones comerciales entre México y Colombia han evolucionado favorablemente a partir de la firma del Tratado de Libre Comercio entre estos países, el 13 de junio de 1994, mismo que entró en vigor el 1 de enero de 1995. Este Tratado se celebró con el carácter de Acuerdo de Complementación Económica (ACE) de acuerdo con lo dispuesto en el Tratado de Montevideo 1980 y en la Resolución No. 2 del Consejo de Ministros de Relaciones Exteriores de las Partes signatarias. El Acuerdo se registró ante la Asociación Latinoamericana de Integración (ALADI) como Acuerdo de Complementación Económica (ACE) No. 33. El Tratado del Grupo de los Tres (TLC-G3), integrado por México, Colombia y Venezuela, se firmó el 13 de junio de 1994 y entró en vigor el 1 de enero de 1995, mediante la Ley de la República de Colombia No. 172 de 1994. Esto quiere decir que originalmente este tratado involucraba también a Venezuela, quien el 22 de mayo de 2006, presenta la denuncia y abandona formalmente el bloque comercial.

A partir de este proceso, para la segunda parte de 2009, Colombia y México acordaron adaptar el TLC suscribiendo cinco decisiones contenidas en un protocolo modificatorio referidas al acceso a mercados, las adecuaciones a las reglas de origen, el Comité Regional de Insumos, las facultades adicionales a la Comisión Administradora y el cambio de nombre del Tratado. Esta profundización del Acuerdo está vigente desde el 2 de agosto de 2011 (Mincomercio, 2019). Por este motivo, el mercado colombiano es una opción comercial internacional para las empresas mexicanas.

Específicamente para el estado de Guanajuato, datos de la Coordinadora de Fomento al Comercio Exterior (COFOCE, 2019), relacionados para el sector exportador de agroalimentos, indican que se obtuvo un $6.6 \%$ de la participación total estatal, esta cifra sólo es superada por las Autopartes (27.6\%).
Para el mercado de las exportaciones hasta octubre de 2018, fueron del orden de 19 mil 210 millones de dólares. 31 de los 46 municipios exportan al mundo, lo que significa un $67 \%$ del comercio exterior total de Guanajuato. Conforme a estas cifras, $22.4 \%$ son micro (MIPE) y $35.2 \%$ son pequeñas (PYME). Se puede afirmar entonces, que Guanajuato posee ventajas comparativas importantes en el sector agroindustrial.

Según datos de Trade Map (2019), el comercio bilateral entre Colombia y México llegó a los 4,838,511 miles de USD en 2017, de los cuales, México exportó hacia Colombia, 3,164,182 miles de USD e importó de Colombia, $1,674,329$ miles de USD. La balanza comercial presenta un superávit de 1,489, 853 en 2017 para México. Por este motivo, al existir el potencial de mercado, la innovación es entendida desde la base de una propuesta mercadológica instrumentada especialmente en la empresa.

\section{Propuesta de internacionalización}

La propuesta de internacionalización está aplicada a una salsa mexicana estilo Bajío, elaborada a base de aceite, ajo, perejil, chile molido y sal, entre otros ingredientes. Tradicionalmente se usa para sazonar carnes rojas, pollo o pescado, aunque también puede aplicarse a ensaladas 0 procesos de maceramiento de alimentos. La gastronomía colombiana es una mezcla de tradiciones culinarias de las civilizaciones prehispánicas que habitaron la región, de la cultura española que la conquisto y de la cultura africana. La razón por la cual se ha elegido la salsa como producto a exportar a Colombia es porque podría encajar fácilmente en la gastronomía colombiana, ya que su potente y penetrante sabor combina perfectamente con las arepas y sanconchos que son peculiarmente populares en este país.

\section{Innovación empresarial}

La Organización para la Cooperación y el Desarrollo Económicos (OCDE, 2018), establece en el Manual de Oslo, que la innovación puede presentarse en las empresas que realizan cambios en sus productos, para también incorporar mejoras en sus procesos de 
comercialización (en este caso relacionado a la internacionalización), lo que llevará a incorporar cambios adicionales a los me método organizativo, en las prácticas internas de la empresa, la organización del lugar de trabajo o las relaciones exteriores. Atendiendo a lo anterior, la mipe puede considerarse como innovadora, a partir de sus cambios para introducir el producto en el mercado colombiano, mismo que implicará mejoras permanentes de sus procesos, operaciones o productos, que impliquen un cambio significativo en alguno de ellos.

\section{Oportunidades según la demanda}

Según la herramienta del Banco Mundial Trade Map (2019), Colombia ocupa el puesto número $57^{\circ}$ de los países que importan los productos englobados en la fracción arancelaria en la que se ubica la salsa.

\section{Importaciones y competencia}

Según Trade Map (2019), El valor total importado es de 24, 773 miles de USD y tiene una tasa de crecimiento anual de $3 \%$ en valor entre 2016 y 2017 . México se encuentra entre los primeros proveedores de Colombia de este tipo de productos, el primer lugar se lo lleva Estados Unidos de América con 12,196 miles de USD, el segundo es México con 8,064 miles de USD de México, tercero es Canadá con 523 miles de USD y cuarto España con 477 miles de USD.

\section{Ambiente financiero Colombia}

Según Datos Macro (2019), dentro de rating que mide la capacidad de un país para hacer frente a su deuda y el riesgo que conlleva para invertir, Colombia se encuentra clasificada de la siguiente manera según las principales agencias de clasificación de riesgo: Tiene un grado medio inferior de capacidad de pago de su deuda.

\section{Ambiente Cultural}

En Colombia y en México predomina el idioma español y la religión católica, como consecuencia de que comparten la historia de la conquista de España sobre su territorio y población autóctona y la colonización. Una característica de los países Latinoamericanos, como Colombia y México, es la concentración de riqueza y de poder en un sector privilegiado de la población. Por otro lado, estas dos culturas se caracterizan por su hospitalidad, amabilidad y un gran sentido del humor. En los negocios Según Legiscomex (2019), a la hora de tomar decisiones la mayoría de los colombianos dejan todo para última hora, pues creen que todo dependerá de la relación personal y de la suerte. Se cree que esta actitud tiene que ver con la cultura de incertidumbre a la que Colombia está habituada.

\section{Sector alimenticio en el país destino: Colombia}

Según la Agencia de Promoción de Inversión para Bogotá Invest in Bogotá (2019), El sector de alimentos en Colombia tiene un alto potencial de desarrollo y se estima que tenga un crecimiento de $39 \%$ aproximadamente entre 2016 y 2021, además de que el país está llevando a cabo nuevas estrategias para la atracción de inversión y modernización del sector. Esto hace de Colombia un mercado meta muy atractivo.

La ciudad colombiana a la que se pretende llevar el producto es Bogotá, hay muchas razones que justifican la elección de esta ciudad, para empezar, es el mercado principal y es el centro de producción de alimentos y bebidas en este país, solo la región Bogotá, tiene el 45\% de las ventas de la industria alimenticia del país, además de que ahí se encuentran 39 de las 100 principales empresas del sector. La ciudad de Bogotá tiene una ubicación geográfica estratégica en Colombia, se puede tener acceso preferencial a los principales mercados de la región. La ciudad cuenta con una gran disponibilidad y calidad de mano de obra. 
Ventas de alimentos procesados en Colombia (2010-2020*)

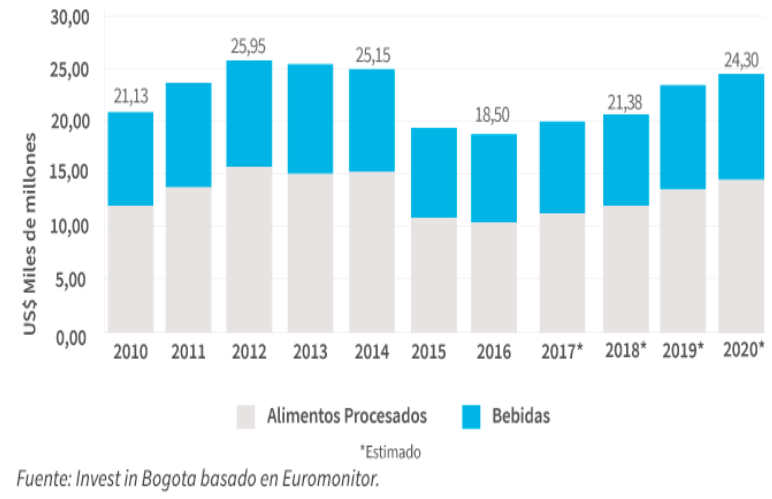

Los colombianos tienen una gran apreciación por la cultura mexicana, como dato curioso, después de México, Colombia es el país que tiene más Mariachis en el mundo. Son fieles seguidores de los dramas de las telenovelas mexicanas y de programas como el Chavo del Ocho, tanto que se han organizado gran cantidad de eventos en este país para homenajearlo. La apreciación de los colombianos por la gastronomía mexicana no podía quedar fuera, en la capital colombiana, Bogotá, según datos del Blog Restorando (2019), hay un gran número de restaurantes mexicanos, por mencionar algunos de ellos.

- La Vecindad. Ubicado en pleno corazón de Bogotá, en el Barrio de poder adquisitivo alto (Chapinero). Este restaurante está inspirado en la serie televisiva El Chavo de Ocho, ofrece parrilladas de carnes y mariscos, aguacate, frijoles, arroz y tortillas.

- Qué Chulo es México. También ubicado en el Barrio de Chapinero, este restaurante ofrece pozole, carne a la tampiqueña, sopa azteca, tacos, enchiladas, agua de jamaica, horchata y tamarindo.

- La Lupita Restorán. Está ubicado en la Zona G1 de la capital colombiana, dentro de su menú, se puede encontrar molcajete de mariscos, tacos de arrachera y bebidas con tequila y mezcal.
${ }^{1}$ La Zona $\mathrm{G}$ es conocida en Bogotá, como la zona gastronómica, punto neurálgico para el consumo de alimentos de alta cocina, por encontrarse cerca del circuito de bancos y comercios en el corazón de la ciudad.
- Plaza México. Ubicado en la exclusiva zona de Hacienda Santa Bárbara, este restaurante ofrece fajitas, carnitas y taquizas.

- Los Cuates. Se encuentra en Los Barrios Unidos, cuenta con una gran variedad de entradas acompañadas de diferentes tipos de salsas y guacamole, el platillo más famoso dentro de su menú son las enchiladas. Aquí se ofrecen fiestas temáticas mexicanas para despedidas empresariales, graduaciones, bodas, aniversarios, fiestas de fin de año y de cumpleaños.

Este tipo de restaurantes serían el mercado meta inicial, ya que, las personas que consumen en ellos son el tipo de clientes que buscan experimentar o deleitarse con la cocina mexicana, abriéndole las puertas, de esta forma, a la salsa mexicana. Una vez que la salsa se encuentre posicionada, sería una buena opción, introducirla en supermercados y tiendas populares en la ciudad de Bogotá.

\section{Tendencias globales de consumo para 2019}

Según el reporte de Euromonitor Internacional (2019), las siguientes son algunas tendencias de consumo que estarán vigentes en el mundo en este 2019 y favorecerán, en este caso, la venta de salsa tipo mexicano en Colombia:

- Tendencia "Quiero un mundo libre de plásticos": En los últimos años, el mundo ha empezado a hacer consciencia del impacto negativo que tiene en el planeta, el uso excesivo de plásticos en los productos de consumo frecuente. En 2019 seguirá creciendo esta preocupación del consumidor por lograr un mundo libre de plástico. Es por eso por lo que sería necesario reemplazar el envase de plástico que actualmente tiene el producto, por un envase de vidrio o biodegradable, de esta forma, se trata de contribuir al bienestar del medio ambiente y hacer felices a los consumidores conscientes sobre esta situación. 
- Tendencia "Regresar a lo básico": Los consumidores rechazan cada vez más los productos genéricos y fabricados en masa. Todos los productos posicionados como más simples, diferentes y auténticos, se verán beneficiados en 2019. Es por esto por lo que el proceso artesanal de la salsa será una ventaja competitiva, pero para que esto suceda, se debe dejar en claro a los consumidores que se trata de una salsa cien por ciento casera y con una receta que ha sido transmitida de generación en generación.

- Tendencia "Digitalmente juntos": La mayoría de las personas se conectan a las redes sociales todos los días y varias veces al día. En 2019, los usuarios de estas plataformas seguirán aumentando. Es indispensable que la empresa cuente con una buena página web, que tenga enlaces a todas las redes sociales de la misma, como Facebook, Instagram y Twitter. De esta forma se asegura una buena comunicación con el consumidor y se genera confianza con el producto. En este caso, se puede aprovechar el interés del mercado colombiano por la gastronomía mexicana y compartir recetas de platillos mexicanos que combinen con la salsa.

\section{METODOLOGÍA}

La presente investigación aborda el enfoque cualitativo desde la perspectiva de estudio de caso relacionado con una mipe agroindustrial de Guanajuato. se presume que las empresas exportadores son más proclives a tener procesos de innovación que las llevan a ser exitosas en mercados internacionales. Se realizó el análisis de las 5 fuerzas de mercado de Porter, y para la investigación del estudio de caso, se aplicó un cuestionario para medir el potencial de la empresa (Arredondo-Hidalgo, 2017). Las áreas consideradas son: $a$. Desarrollo del Producto, $b$. Eficiencia en la producción, c. Mercadotecnia Internacional y d. Gestión Gerencial. En la última parte del cuestionario, se adicionaron 13 ítems relacionados a medir el grado de innovación empresarial.

\section{Contexto del estudio de caso.}

Es una empresa familiar pequeña que opera en el municipio de Irapuato, Gto., desde el año 2000. Actualmente, cuenta con un total de 20 trabajadores, cada uno de ellos son profesionales y especialistas en su área de trabajo, 14 de ellos son ayudantes o chefs secundarios, 3 se encuentran a cargo del área administrativa de la empresa, 2 se encuentran en el área de ventas/marketing y una persona es la encargada de la dirección general y es dueña de la empresa. Todos los insumos para la elaboración del producto cuentan con la más alta calidad, además de que se tiene una línea de productos bajos en calorías. Los aderezos se venden sobre pedido a los clientes que procesan o brindan servicios de alimentos, además de que existe una pequeña tienda en la cual se ofrecen los productos al público en general.

\section{Valor estratégico del negocio}

Porter (2008) acuñó el concepto de ventaja competitiva del mercado, relacionando cinco fuerzas bajo las cuales debe visualizarse el negocio, siempre identificando, tanto las oportunidades de inversión y crecimiento, desde la inversión que la empresa dirige. La siguiente tabla identifica las 5 fuerzas analizadas para el mercado de exportación en Colombia. 
ISSN: 1794-9920 Impreso / Electrónico 2500-9338

Agosto - Diciembre. Volumen 19 Número 2, Año 2019 Págs. 104-114
Tabla 1.

Análisis del modelo de Porter para el mercado colombiano

\begin{tabular}{|c|c|}
\hline Fuerzas & Análisis \\
\hline $\begin{array}{l}\text { 1. Poder de } \\
\text { negociación de los } \\
\text { clientes }\end{array}$ & $\begin{array}{l}\text { Específicamente la ciudad de Bogotá, existen un } \\
\text { gran número de restaurantes, mismos que son } \\
\text { considerados como mercado meta. Existe una } \\
\text { gran posibilidad de hacer una gran cartera de } \\
\text { clientes con este tipo de restaurantes, una vez } \\
\text { que el producto esté más posicionado en el } \\
\text { mercado, se podrá pensar en la idea de } \\
\text { venderla directamente a los consumidores } \\
\text { finales como amas de casa o cocineros del } \\
\text { último canal de consumo. }\end{array}$ \\
\hline $\begin{array}{l}\text { 2. Naturaleza de la } \\
\text { Rivalidad de la } \\
\text { competencia }\end{array}$ & $\begin{array}{l}\text { En la ciudad de Bogotá, al ser una zona famosa } \\
\text { por su gastronomía, existen varios proveedores } \\
\text { de salsas y aderezos, lo cual no necesariamente } \\
\text { representa una fuerte amenaza para el } \\
\text { producto, dado que la salsa tiene un proceso de } \\
\text { producción diferenciado. Una gran ventaja es } \\
\text { que los consumidores suelen visitar Bogotá con } \\
\text { la intención de probar platillos nuevos y } \\
\text { exóticos, lo cual minimiza los riesgos para } \\
\text { nuestra salsa tipo mexicano. }\end{array}$ \\
\hline $\begin{array}{l}\text { 3. Amenaza de } \\
\text { nuevos competidores } \\
\text { entrantes }\end{array}$ & $\begin{array}{l}\text { El mercado colombiano de exportación reviste la } \\
\text { facilidad de que nuevos competidores sean } \\
\text { parte del contexto, pues al existir tantos } \\
\text { restaurantes en esta zona, los competidores } \\
\text { entrantes ven en ellos una oportunidad de } \\
\text { negocio por considerarlos a todos clientes } \\
\text { potenciales. Necesariamente esto puede } \\
\text { identificarse como ventaja y desventaja a la vez. } \\
\text { Para la etapa de penetración del mercado meta, } \\
\text { esto representa una ventaja, dada la } \\
\text { diferenciación del producto. No obstante, ya que } \\
\text { se cuente con una participación en el mercado } \\
\text { colombiano, otros productores podrían procesar } \\
\text { alimentos parecidos al original. }\end{array}$ \\
\hline $\begin{array}{l}\text { 4. Poder de } \\
\text { negociación de los } \\
\text { proveedores }\end{array}$ & $\begin{array}{l}\text { La empresa guanajuatense exportaría la salsa } \\
\text { completamente preparada hasta Colombia, no } \\
\text { existiría una relación directa con los } \\
\text { proveedores, a menos de que los insumos de } \\
\text { preparación sean más baratos en Colombia que } \\
\text { en México, entonces se podría analizar la } \\
\text { cadena de valor, trasladando la producción de } \\
\text { Guanajuato a Colombia. Por consiguiente, no se } \\
\text { tendría un nivel de dependencia al tener } \\
\text { proveeduría local para el proceso productivo de } \\
\text { origen. }\end{array}$ \\
\hline $\begin{array}{l}\text { 5. Amenaza de } \\
\text { productos sustitutos }\end{array}$ & $\begin{array}{l}\text { Por ser un producto de consumo sectario, y al } \\
\text { tratarse de un aditivo, el producto presenta una } \\
\text { variedad amplia de sustitutos, dado que } \\
\text { cualquier otro tipo de alimento podría sustituirla } \\
\text { a la hora de acompañar los alimentos, pero no } \\
\text { existe otra salsa parecida a esta en el mercado } \\
\text { colombiano, la diferenciación puede ayudar } \\
\text { mucho, pues al ser una salsa mexicana, cuenta } \\
\text { con un alto valor agregado, en Colombia, los } \\
\text { restaurantes mexicanos están de moda y al } \\
\text { promocionarla como mexicana, puede que la } \\
\text { haga más atractiva para el consumidor. }\end{array}$ \\
\hline
\end{tabular}

\section{Fracción Arancelaria y Regulaciones y Restricciones No Arancelarias}

El objetivo de este proyecto es el analizar la factibilidad de exportar la salsa de México a Colombia. Los aranceles se aplican a todos los productos, basados en el Sistema Armonizado, sin embargo, estas tasas tienden a disminuirse con el tiempo. Desde el $1^{\circ}$ de enero de 1995, entró en vigor el Tratado del grupo de los Tres (TLC-G3), entre México, Colombia y Venezuela, tiempo después, sin efecto con Venezuela. Hoy en día, más del $97 \%$ de los productos tiene un arancel del $0 \%$. Para este caso, la fracción arancelaria para la salsa coincide en las tarifas de ambos países.

\section{Fracción arancelaria: México}

Según la Ley de los Impuestos Generales de Importación y Exportación (LIGIE) consultada en CAAAREM (2019), la fracción arancelaria que corresponde a la salsa es: 21.03.90.99. La unidad de medida es en kilogramos.

\begin{tabular}{|c|c|}
\hline Sección: IV & $\begin{array}{l}\text { Productos de las industrias } \\
\text { alimentarias; } \\
\text { alcohólicos y vebidas, líquidos } \\
\text { sucedáneos del tabaco elaborado }\end{array}$ \\
\hline Capítulo: 21 & Preparaciones alimenticias diversas \\
\hline Partida: 2103 & $\begin{array}{l}\text { Preparaciones para salsas y salsas } \\
\text { preparadas; condimentos y sazonadores, } \\
\text { compuestos; harina de mostaza y mostaza } \\
\text { preparada. }\end{array}$ \\
\hline $\begin{array}{l}\text { Subpartida: } \\
210390\end{array}$ & Los demás. \\
\hline $\begin{array}{l}\text { Fracción } \\
\text { arancelaria: } \\
21.03 .90 .99\end{array}$ & Los demás. \\
\hline
\end{tabular}

Esta fracción arancelaria, paga un IVA del 19\% a la importación, sin embargo al importar en Colombia de México, los productos englobados en esta fracción arancelaria pagarán $0 \%$ de gravamen arancelario. Los documentos soporte para la importación de esta fracción arancelaria en Colombia son: Registro Sanitario y visto bueno del Instituto Nacional de Vigilancia de Medicamentos y Alimentos mediante el trámite previo. 


\section{Documentos para la exportación en México}

Según el Artículo 36 de la Ley Aduanera, quienes introduzcan y extraigan mercancías del territorio nacional destinándolas a un régimen aduanero, están obligados a presentar la siguiente documentación (ProMéxico, 2019):

- Factura comercial

- Encargo conferido (autorización ante la autoridad aduanera para que el agente aduanal lleve a cabo el despacho a nombre del exportador)

- Carta de instrucciones al agente aduanal (información específica y detallada de la operación y se entrega directamente al agente aduanal)

- Lista de empaque

- Certificado de origen

- Documento del transporte

- Documentos que avalen el cumplimiento de las regulaciones y restricciones no arancelarias, tales como: certificados sanitarios, certificados de calidad, permisos, etc.

\section{Documentos para la importación en Colombia}

Según datos de la Cámara de Comercio de Bogotá (2017), los siguientes documentos se necesitan para importar en Colombia:

- Factura comercial emitida por el exportador.

- Registro o licencia de importación (si requiere) con el que se acreditarán los permisos, requisitos y autorizaciones a que haya lugar.

- La prueba de origen comercial (si se requiere), para la aplicación de disposiciones especiales 0 tratamientos preferenciales.

- Lista de empaque. (si requiere)

- Documento de transporte: aéreo (guía aérea), marítimo (conocimiento de embarque) y terrestre (carta porte).
- Declaración andina de valor (cuando la importación supere los USD5.000 en un solo envío 0 en envíos fraccionados que sumen USD5.000)

- Mandato cuando la declaración de presente a través de una agencia aduanera.

\section{Logística Internacional.}

Dado que el objetivo es llevar la salsa desde Guanajuato hasta Bogotá, Colombia, la mejor opción es trasladar el producto en transporte marítimo, ya que es más económico en comparación a otros tipos de transporte. Irapuato en Guanajuato es la ciudad mexicana en donde se encuentra ubicada la empresa productora. El Puerto de Manzanillo, Colima, es el puerto mexicano por cual saldrá el producto, este puerto es uno de los más accesibles para la zona centro de México. El Puerto de Buenaventura, Valle del Cauca es el puerto colombiano al cual arribará el producto, este puerto es el más importante de Colombia, además de que es el más cercano a la ciudad destino del producto, Bogotá, Distrito Capital.

\section{RESULTADOS:}

A partir del cuestionario aplicado para analizar el potencial exportador de la empresa, la tabla siguiente muestra los resultados obtenidos para la mipe guanajuatense. 


\begin{tabular}{|cccc|}
\hline $\begin{array}{c}\text { Variables Potencial } \\
\text { Exportador }\end{array}$ & Porcentaje ideal & Promedio obtenido & Porcentaje total \\
\hline Desarrollo del producto & $40 \%$ & 3.8182 & 1.12 \\
\hline Eficiencia de la producción & $25 \%$ & 4.2353 & 1.05 \\
\hline $\begin{array}{c}\text { Estructura comercial y de } \\
\text { mercadotecnia } \\
\text { internacional }\end{array}$ & $20 \%$ & 3.1429 & 0.6286 \\
\hline Gestión gerencial & $15 \%$ & 2.7143 & 0.4071 \\
\hline Total & $100 \%$ & $=$ & 3.2218 \\
\hline${ }^{\text {Innovación }}{ }^{1}$ & 13 ítems & Calificación global obtenida: 4.15 \\
\hline
\end{tabular}

El promedio total obtenido es de 3.22 , de un puntaje máximo de 5. Eso quiere decir que la empresa, es capaz de llevar el producto al mercado colombiano, aunque le hace falta mejorar en algunas áreas para poder internacionalizarse con los menores riesgos posibles.

La variable más robusta es la relacionada a la eficiencia de la producción dato que se obtuvo un 4.23 de un máximo de 5. Entonces la empresa es muy eficiente en los procesos de calidad y de producción, lo que es sin duda positivo en relación a los estándares de normas de calidad y de adaptarse a nuevos pedidos que le podrán resultar en escalar la oferta exportable. Esta variable se relaciona también al puntaje obtenido para el considerado como Desarrollo del Producto, donde la calificación fue de 3.81, lo que se traduce en que la administración de los ingredientes de la salsa está coordinados y la administración del empaque y sus adaptaciones son idóneos para el mercado colombiano.

La variable Estructura Comercial y de Mercadotecnia Internacional, obtuvo un 3.14 de un máximo de 5, este puntaje sigue siendo bueno, aunque más bajo que el de los dos anteriores, la empresa se encuentra bien en esta área, pero si quiere exportar con éxito no será suficiente, deberá atender a profundidad los aspectos relacionados con el plan de internacionalización, involucrarse con este proceso y de ser posible reclutar especialistas en el área a la vez de capacitar a su personal actual.
Finalmente, la calificación más débil fue para la variable Gestión gerencial, con un puntaje de 2.71 de un máximo de 5 , al tratarse de una MIPE, deberá ser más eficiente en la organización de los procesos administrativos, lo que representa un reto, si la empresa persigue el éxito, tanto en el mercado nacional, como en el internacional.

\section{CONCLUSIONES}

Se elige el mercado colombiano, específicamente para el distrito de Bogotá, por tener el antecedente del Tratado comercial y porque la comida mexicana es muy apreciada en este punto comercial. La información de datos y consumo se ha hecho a partir de la herramienta del Centro de Comercio Mundial relacionada directamente al Trade Map, así como de requerimientos legales para llegar al mercado de Colombia.

Con base en la investigación presentada, se concluye que el mercado colombiano es una opción potencial de internacionalización de la empresa. Sin embargo, es necesaria a la adaptación para este mercado, esto se traduce como mejoras que tendrían que aplicarse al producto, para hacerla mucho menos picante, porque a ninguna otra cultura en el mundo, le gusta experimentar esa sensación de picor como a los mexicanos y los colombianos no son la excepción.

Asimismo, es probable que la salsa estilo Bajío tenga que adaptar otro estilo de imagen en el mercado colombiano, respecto al diseño del empaque, envase y etiquetas. Como 
se mencionó con anterioridad, lo que le abrirá las puertas del mercado colombiano al producto, es evidenciarlo como cien por ciento mexicano. La empresa necesita diseñar una etiqueta enfocada a que el consumidor se de cuenta que es un producto mexicano, también sería recomendable sustituir el envase de plástico por uno de vidrio para lograr una apariencia más estética, y considerando aspectos de sustentabilidad y cuidado del medio ambiente.

Otro aspecto no menos importante, es el de hacer más grande la oferta exportable, aumentando la capacidad productiva para hacer frente su problemas a pedidos mayores y más constante, es necesario contar con cuotas de venta como objetivos comerciales inmediatos para el mercado colombiano.

La solvencia económica es una condición para que se considere el éxito en los planes de internacionalización de la MIPE. Sin embargo, no se recomienda una inversión alta al comienzo de los planes de internacionalización. No obstante, de lo anterior, otra forma de concretar el éxito fuera de México es que la mipe tenga alianzas comerciales para expandirse paulatinamente, tales como representaciones de distribución o licencias de marca que les permita fortalecer la presencia de la marca en el mercado de bogotano. A largo plazo, las cadenas de valor podrían incorporarse estratégicamente, al analizar si la producción pueda trasladarse, para cubrir la totalidad del mercado hacia otros distritos de Colombia.

\section{REFERENCIAS:}

Arredondo-Hidalgo, M.G. (2017). Cuestionario para medir el potencial exportador.

CAAAREM. (2019). Tarifa de la ley de impuestos generales de importación y exportación. Recuperado de https://www.siicex-caaarem.org.mx/

Cámara de Comercio de Bogotá (2017). Requisitos para importar en Colombia. Recuperado el 07 de febrero de 2019, de https://bibliotecadigital.ccb.org.co/bitstream/handl e/11520/14384/Gu\%C3\%ADa\%20Pr\%C3\%A1ctic a \%20Proceso\%20de \%20Importaci $\%$ C3\%B3n $\% 2$ Ode $\% 20$ Bienes. . dff?sequence $=3$ \&isAllowed $=y$

Coordinadora de Fomento al Comercio Exterior de Guanajuato [COFOCE] (2019). Boletín Innovar para competir en el mundo. (2019). Recuperado de:

https://www.yumpu.com/es/document/read/62389 $\underline{091 / \text { gto-up-5 }}$

Datos Macro. (2019). Rating: Calificación de la deuda de los países. Recuperado de: https://datosmacro.expansion.com/ratings

Dirección de Impuestos y Aduanas Nacionales. (2019). Estructura arancelaria. Recuperado de: https://muisca.dian.gov.co/WebArancel/DefConsu ItaEstructuraArancelaria.faces

Euromonitor Internacional. (2019). Las 10 principales tendencias globales de consumo para 2019. Recuperado de https://www.euromonitor.com/

Gallardo-Vázquez, Dolores, Sánchez-Hernández, M. I. y Corchuelo-Martínez-Azúa, M.B. (2013). Validación de un instrumento de medida para la relación entre la orientación a la responsabilidad social corporativa y otras variables estratégicas de la empresa. Revista de contabilidad. Spanish accounting review 16(1), 11-23.

Gobierno de Colombia. Mincomercio. (10 de febrero de 2019). Tratado de Libre Comercio con México. Recuperado de: http://www.tlc.gov.co/publicaciones/11963/tratado _de_libre_comercio_entre_los_estados_unidos mexicanos_y_la_republica_de_colombia

Invest in Bogotá. (2019). Alimentos y bebidas. Recuperado de: https://es.investinbogota.org/sectores-deinversion/alimentos-y-bebidas-en-bogota

OECD. (2018). Oslo Manual. Guidelines for Collecting, Reporting and Using Data on Innovation, 4th. Edition. Recuperado de: http://www.oecd.org/science/oslo-manual-2018$\underline{9789264304604-e n . h t m}$

Legiscomex. (2019). Cultura de negocios con Colombia. Recuperado el 09 de febrero de 2019, de 
ISSN: 1794-9920 Impreso / Electrónico 2500-9338

Agosto - Diciembre. Volumen 19 Número 2, Año 2019 Págs. 104-114

https://www.legiscomex.com/Documentos/culturanegocios-colombia-rci308

Porter, M. (2008). Estrategia Competitiva ( $38^{\circ}$ Ed.). México, D.F., México: Grupo Editorial Patria.

ProMéxico. (2019). Documentos y requisitos generales para exportar. Recuperado de: http://www.promexico.gob.mx/es/mx/pasosexportar

ProColombia. (2019). Rutas Marítimas. Recuperado el 09 de febrero de 2019, de http://www.colombiatrade.com.co/herramientas/lo gistica/reportes-de-transporte/maritimo/rutas

Restorando. (2019). Restaurantes cocina mexicana en Bogotá. Recuperado de: https://bogota.restorando.com.co/restaurantescocina-mexicana

Thompson, A., Strickland, A. y Gamble, J. (2008). Administración Estratégica: Teoría y casos $\left(15^{\circ}\right.$ Ed.). México, D.F., México: Mac Graw Hill Interamericana.

Trade Map. (2019). Lista de los países importadores para el producto seleccionado en 2017 Producto: 210390 Preparaciones para salsas y salsas preparadas; condimentos y sazonadores, compuestos. Recuperado el 07 de febrero de 2019, de https://www.trademap.org/Country_SelProduct.as px?nvpm $=3 \% 7 \mathrm{c} \% 7 \mathrm{c} \% 7 \mathrm{c} \% 7 \mathrm{c} \% 7 \mathrm{c} 2103 \% 7 \mathrm{c} \% 7 \mathrm{c} \%$ 7c4\%7c1\%7c1\%7c1\%7c1\%7c\%7c2 $\% 7 \mathrm{c} 1 \% 7 \mathrm{c} 1$

Trade Map. (2019). Lista de los mercados proveedores para un producto importado por Colombia en 2017 Producto: 210390 Preparaciones para salsas y salsas preparadas; condimentos y sazonadores, compuestos. Recuperado el 07 de febrero de 2019, de https://www.trademap.org/Country_SelProductCo untry.aspx?nvpm $=3 \% 7 \mathrm{c} 170 \% 7 \mathrm{c} \% 7 \mathrm{c} \% 7 \mathrm{c} \% 7 \mathrm{c} 210$ $3 \% 7 \mathrm{c} \% 7 \mathrm{c} \% 7 \mathrm{c} 4 \% 7 \mathrm{c} 1 \% 7 \mathrm{c} 1 \% 7 \mathrm{c} 1 \% 7 \mathrm{c} 1 \% 7 \mathrm{c} \% 7 \mathrm{c} 2$ \%7c1\%7c1 